\title{
Desempeño satisfactorio de la Planta de Irradiación Gamma en el ensayo de aptitud, programa INTERLAB N-DO-02
}

\section{Satisfactory performance of the Gamma Irradiation Plant on program N-DO-02 of the INTERLAB proficiency test}

Recibido: 16 de octubre de 2019

Aceptado: 19 de diciembre de 2019

Publicado en línea: 30 de diciembre de 2019

Doi: https://doi.org/10.32685/2590

7468/invapnuclear.3.2019.511

Palabras clave: ensayos de aptitud, comparaciones interlaboratorio, dosis de radiación, aseguramiento de la calidad.

Keywords: proficiency testing, interlaboratory comparisons, radiation dose, quality assurance

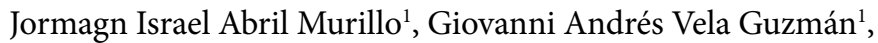
Azarías de Jesús Moreno Machado ${ }^{1}$

Citación: J. I. Abril Murillo, G. A. Vela Guzmán y A. J. Moreno Machado, “Desempeño satisfactorio de la Planta de Irradiación Gamma en el ensayo de aptitud, programa INTERLAB N-DO-02", Revista Investigaciones y Aplicaciones Nucleares, n. ${ }^{\circ}$ 3, pp. 40-48, 2019. https://doi.org/10.32685/2590-7468/ invapnuclear.3.2019.511

\section{RESUMEN}

La Planta de Irradiación Gamma del Servicio Geológico Colombiano, única en el país, participó en el programa Ensayo De Aptitud por Comparaciones Interlaboratorio, N-DO-02 dirigido por la División Gestión de Interlaboratorios (INTERLAB) de la Comisión de Energía Atómica de Argentina, como parte fundamental del proceso de aseguramiento de la calidad relacionado con los procesos de irradiación y de dosimetría de rutina propios de la instalación. Los resultados fueron satisfactorios para todos los valores de dosis asignados en el ensayo de aptitud.

\section{ABSTRACT}

The Colombian Geological Survey's Gamma Irradiation Plant, the only one in the country, participated in Interlaboratory Proficiency Comparison Test program N-DO-02, directed by the Interlaboratory Management Division (INTERLAB) of the Atomic Energy Commission of Argentina, as an essential part of quality assurance related to the facility's irradiation and routine dosimetry processes. The results were satisfactory for all dose values assigned in the proficiency test.

${ }^{1}$ Planta de Irradiación Gamma, Dirección de Asuntos Nucleares, Servicio Geológico Colombiano

Email de correspondencia: jabril@sgc.gov.co 


\section{INTRODUCCIÓN}

L os ensayos de aptitud son, en términos generales, una herramienta de aseguramiento de la calidad que permite a los laboratorios comparar su desempeño con sus pares, detectar tendencias y, consecuentemente, tomar cualquier acción preventiva o correctiva necesaria para asegurar su competencia técnica y mejora continua [1]-[3].

Pueden ser de dos tipos [4]:

» Programa de comparación de mediciones

\) Programa de comparaciones interlaboratorio

El primer tipo es más utilizado para evaluar el desempeño de patrones de calibración, en tanto que el segundo tipo es utilizado comúnmente por diferentes laboratorios de ensayo para demostrar su competencia, y será el que se tratará en este documento.

En esta evaluación, de manera complementaria y paralela a las actividades propias del ensayo, se establece una valoración real del nivel de implementación del sistema de gestión, que en este caso se aplica a la Planta de Irradiación Gamma del Servicio Geológico Colombiano (SGC).

A nivel institucional, el Servicio Geológico Colombiano cuenta con un sistema integrado de gestión fundamentado, entre otras normas, en la norma ISO 9001:2015 [5]. Los lineamientos de la norma ISO/IEC 17025:2005 [6] rigen de modo transversal en las distintas instalaciones de la Dirección de Asuntos Nucleares (DAN). En su conjunto, la implementación se encuentra entre un $85 \%$ y $95 \%$, y su objeto es acreditar el método de ensayo de la dosimetría de rutina propia de la instalación.

El programa de Ensayo de Aptitud de la División de Gestión de Interlaboratorios (INTERLAB) N-DO-X fundamenta sus criterios de evaluación en lo establecido por la norma ISO/IEC 17043:2014, "Evaluación de la conformidad: requisitos generales para ensayos de aptitud". A pesar de que el alcance de la acreditación es solo para la "Determinación del contenido de uranio en agua”, el sistema de gestión acreditado es transversal a todos los ensayos que se trabajan en INTERLAB, lo que valida el ejercicio ante la Organización de Acreditación Argentina (OAA) [7] y por diferentes acuerdos de reconocimiento mutuo internacional ante el Organismo Nacional de Acreditación de Colombia (ONAC) [8].
En el ensayo se participó junto con más de diez instalaciones de países latinoamericanos, cuya fuente de irradiación es exclusivamente del tipo de emisores gamma, pues en este caso no se realizó el ejercicio con irradiadores de haz de electrones o de rayos $\mathrm{X}$.

A continuación, se describen los resultados obtenidos por la planta de irradiación en el ejercicio ensayo de aptitud programa N-DO-02.

\section{MÉTODO EXPERIMENTAL}

El ejercicio se realizó atendiendo todas las instrucciones establecidas por INTERLAB en el programa "FO-Interlab-30 RV.12, Programa N-DO-02 junio 2018” [3], así como los lineamientos procedimentales internos de la Planta de Irradiación Gamma del Servicio Geológico Colombiano. El mismo se desarrolla a partir de la evaluación de dos aspectos:

\) Primera parte: La capacidad de los participantes para cumplir con valores de dosis preestablecidos (dosis objetivo), comparando el resultado de cada participante con el valor asignado, utilizando límites determinados por la variabilidad de los resultados de todos los participantes.

\) Segunda parte: La capacidad de los participantes para medir las dosis efectivamente aplicadas. Se realiza al comparar el resultado de cada participante con el valor asignado, utilizando la propia incertidumbre de medición del participante.

\subsection{Materiales}

El proceso de irradiación gamma fue llevado a cabo en el irradiador panorámico tipo IV de Cobalto-60 $\left({ }^{60} \mathrm{Co}\right)$ de almacenamiento de la fuente en húmedo [9], perteneciente al SGC.

El sistema dosimétrico de rutina empleado por la Planta de Irradiación es el de polimetilmetacrilato (PMMA), basado en la norma ASTM 51276-96 [10], tanto en los dosímetros tipo Amber 3042 Perspex, para dosis de $1 \mathrm{kGy}$ a $30 \mathrm{kGy}$, y Red 4034 Perspex, para dosis de 5 kGy a 50 kGy.

Los dosímetros de referencia de alanina son del tipo pellet lote BF 616 y fueron remitidos por la Comisión Nacional de Energía Atómica e INTERLAB (Centro Atómico de Constituyentes, Buenos Aires, Argentina), cada pastilla en un recipiente individual de plástico de poliestireno transparente de alta densidad (figura 1). 
Se utilizan las curvas de calibración (Absorbancia vs Dosis) para los lotes de dosímetros de rutina a evaluar dadas por el fabricante Harwell

Se utilizó el espectrofotómetro Genesys 20 de ThermoScientific $^{\circ}$, instrumento capaz de determinar la absorbancia óptica en la longitud de onda del análisis. Para asegurar el control analítico instrumental y el rendimiento de los diferentes parámetros del sistema espectrométrico se realiza una verificación de rutina, en el marco de aseguramiento de la calidad analítica, empleando un juego de filtros estándar Spectronic, trazables a NIST, para los parámetros:

»Energía radiante difusa en la longitud de onda de 340 $\mathrm{nm}$ y $400 \mathrm{~nm}$

» Transmitancia en $0 \%$

\ Exactitud de absorbancia en $10 \% \mathrm{~T}$ y $50 \% \mathrm{~T}$ a $590 \mathrm{~nm}$

» Exactitud de longitud de onda en 527,4 nm

Se midió el espesor de cada pellet de PMMA con un micrómetro digital de exteriores NSK-Digitrix Mark III, verificado previamente con un bloque patrón Mitutoyo de 3 $\mathrm{mm}$ de espesor, en el marco de aseguramiento de la calidad analítica (figura 2).

La temperatura a la cual se realizó la irradiación de los ítems de ensayo se determinó por medio de un termómetro digital de punzón calibrado, midiendo inmediatamente antes y después del proceso de irradiación (figura 3).

El personal responsable del proceso de irradiación construye el fantoma conforme a la descripción dada en la norma ISO/ASTM 51261, "Guía estándar para la selección y calibración de sistemas dosimétricos para procesos de radiación" [11], que son las mismas especificaciones del fantoma descritas en el programa N-DO-02. Este se construyó con láminas de polimetilmetacrilato (PMMA) de $1 \mathrm{~cm}$ de espesor y con una tapa hermética, para garantizar equilibrio electrónico dentro del mismo y asegurar que no existieran diferencias significativas en las dosis absorbidas entre los dosímetros insertados (figura 4).

Se utiliza una curva de dosis (kGy) vs. distancia $(\mathrm{cm})$ (gráfica 1), construida con datos experimentales recolectados previamente con dosimetría de rutina y desarrollada en el eje perpendicular al centro geométrico de la fuente radiactiva, desde la superficie de contacto con la lámina de aluminio hasta $50 \mathrm{~cm}$ de distancia, la cual se ajusta con base en el decaimiento de la fuente, y es propia de la operación.

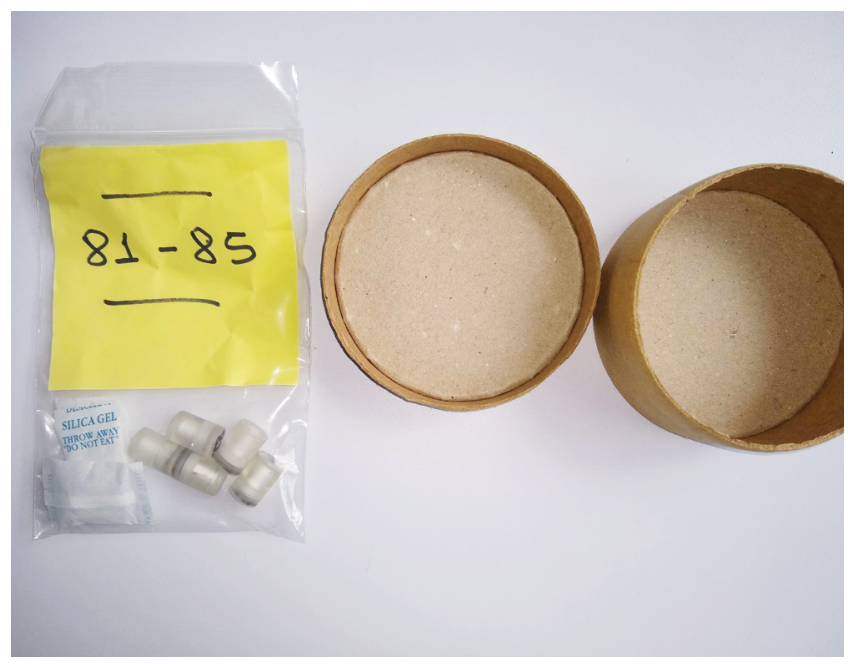

Figura 1. Sistema de dosímetros remitido por INTERLAB

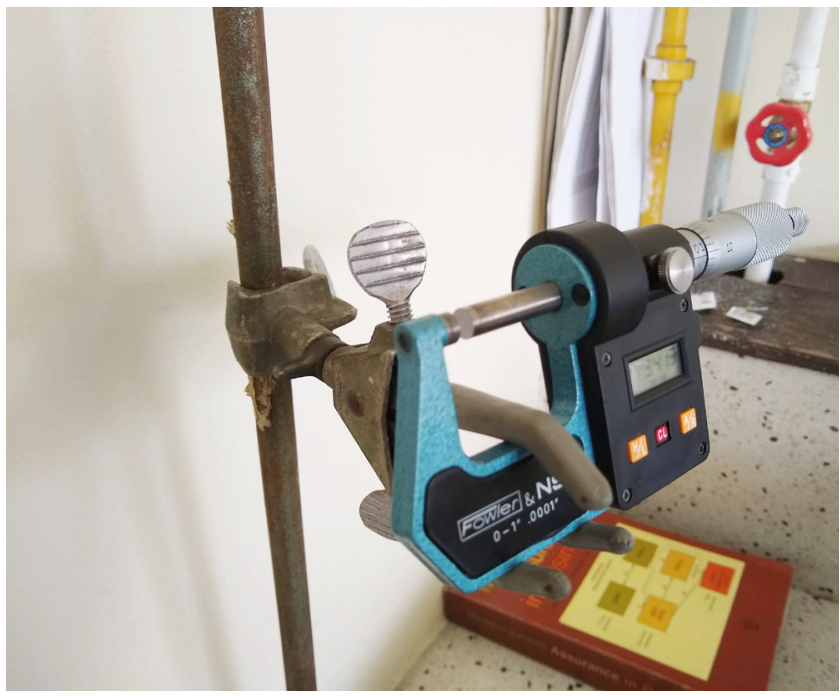

Figura 2. Micrómetro de precisión utilizado en el ensayo de aptitud.

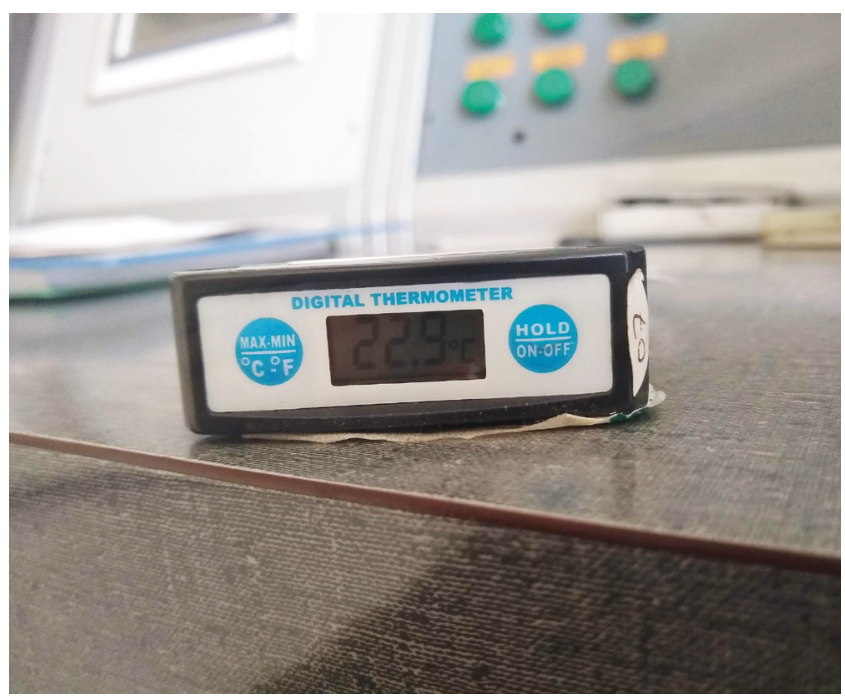

Figura 3. Termómetro de punzón utilizado en el ensayo de aptitud. 


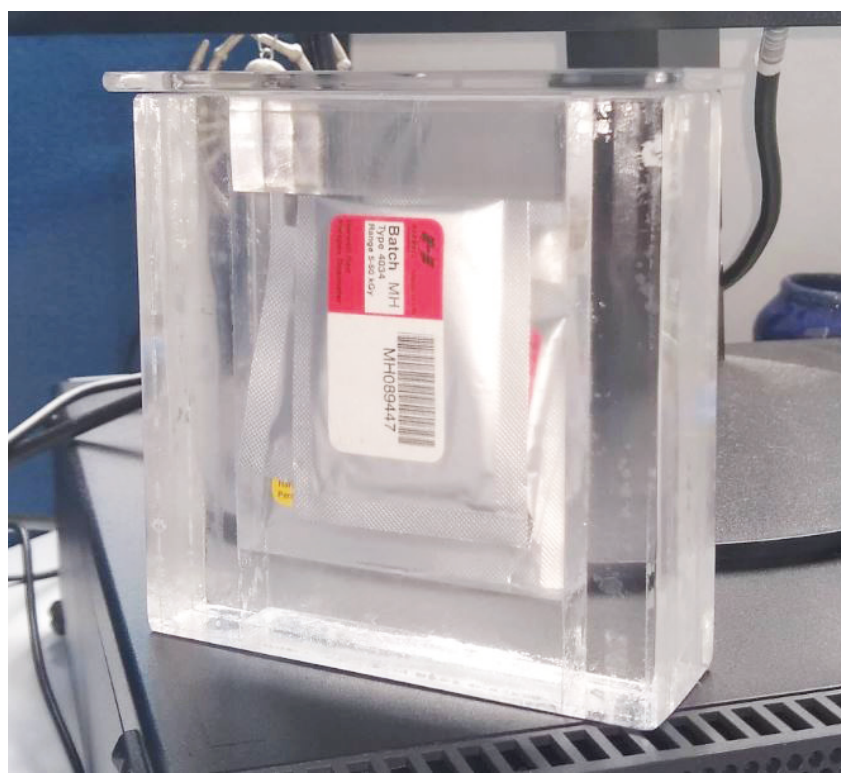

Figura 4. Fantoma construido por el personal para irradiación gamma de los dosímetros.

La captura de datos se realizó de manera digital, mediante formatos que corresponden a los empleados con el método de ensayo, de conformidad con la evaluación de necesidades identificadas durante el desarrollo del ensayo de aptitud.

\subsection{Condiciones ambientales}

Los ítems de ensayo se mantuvieron sellados y protegidos de la luz del sol en el empaque mostrado en la figura 1, a temperaturas por debajo de $\operatorname{los} 25^{\circ} \mathrm{C}$ y humedad controlada con bolsas de desecante, mientras permanecieron bajo la custodia de la Planta de Irradiación. Las dosis de rayos X recibidas en las inspecciones aduaneras no afectaron significativamente la información de las dosis de rayos gamma aplicadas en el ejercicio de intercomparación.

\subsection{Parámetros que se midieron}

Los organizadores del programa de intercomparación evalúan las dosis entregadas a los dosímetros de alanina con radiación gamma y las incertidumbres $\mathrm{U}\left(\mathrm{x}_{\mathrm{pt}}\right)$ asociadas a cada valor objetivo. Las dosis objetivo son $\mathrm{x}_{\mathrm{pt}}$ : [1 kGy, $5 \mathrm{kGy}, 10$ kGy y 25 kGy].

\subsection{Protocolo para el proceso de irradiación}

\subsubsection{Selección de la tasa de dosis}

Siguiendo los lineamientos identificados en [12], se eligió aplicar las dosis definidas a una tasa de 1,5 Gy/s (5,4 kGy/h), valor que está en el rango de tasas de dosis usadas en el es-

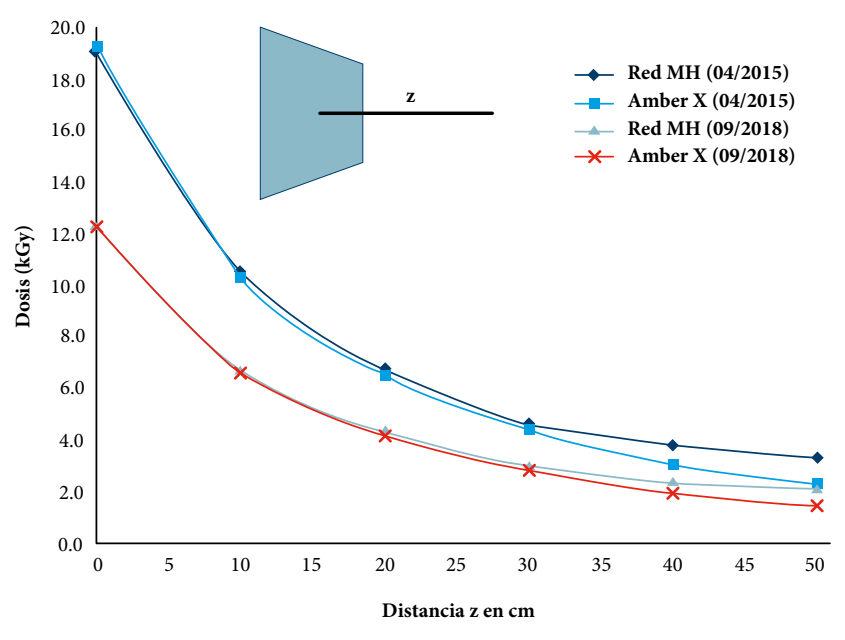

Gráfica 1. Perfiles de dosis experimental absorbida y calculada en la fecha del ensayo con dosímetros PMMA, correspondiente al eje transversal a la fuente radiactiva $(\mathrm{z})$. La distancia $0 \mathrm{~cm}$ corresponde al punto de contacto con la barrera protectora de aluminio.

tudio del mismo autor y es una de las tasas utilizadas por Harwell para calibrar los dosímetros de PMMA en condiciones normales $\left(20^{\circ} \mathrm{C}\right)$.

Por otro lado, el irradiador podía impartir dicha tasa a $15 \mathrm{~cm}$ del centro geométrico de la fuente, medidos perpendicularmente desde la barrera de protección de aluminio. Dicha distancia equivale a la del centro de una caja estándar de producto que pueda ser ubicada en el sistema de transporte, $y$ es representativa de las condiciones normales de producción.

Este dato experimental se obtuvo con el perfil de dosis en el mismo eje perpendicular a la fuente radiactiva, el cual se ajustó con base en el decaimiento alcanzado justo el día del ensayo (gráfica 1).

\subsubsection{Montaje del fantoma}

Según cuál sea la tasa de la dosis requerida, descrita en el numeral anterior, se diseña un montaje (detallado en la figura 5) para garantizar, durante los diferentes ensayos que se realizarán, la repetibilidad en las condiciones de operación, para una ubicación constante del fantoma con los sistemas dosimétricos en el espacio del irradiador.

\subsubsection{Estimación de los tiempos de irradiación}

Experimentalmente se ha configurado un área geométrica sobre la superficie de la lámina de la barrera de aluminio, en donde, con los años de experiencia, se ha identificado un área con la mayor contribución de dosis, que es el área 


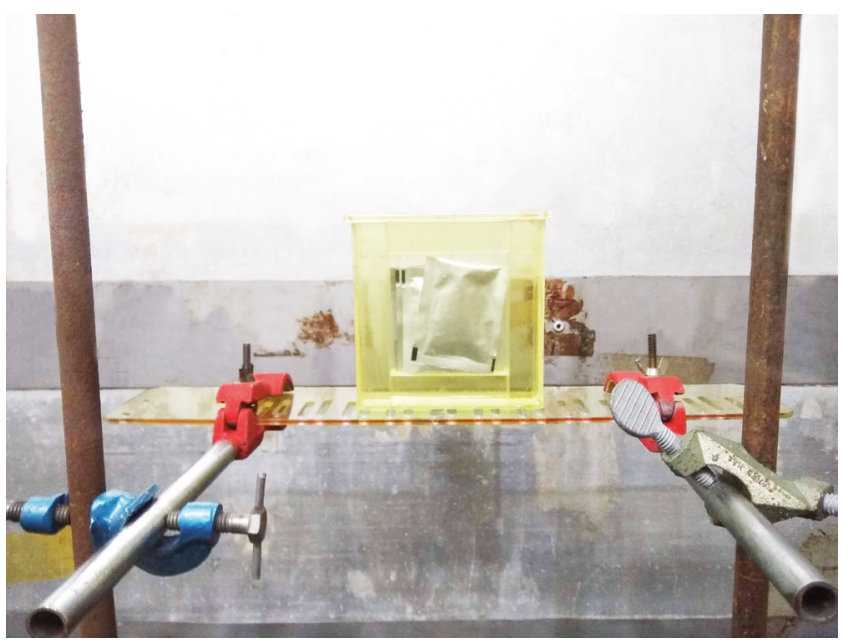

Figura 5. Montaje para irradiar los ítems de ensayo.

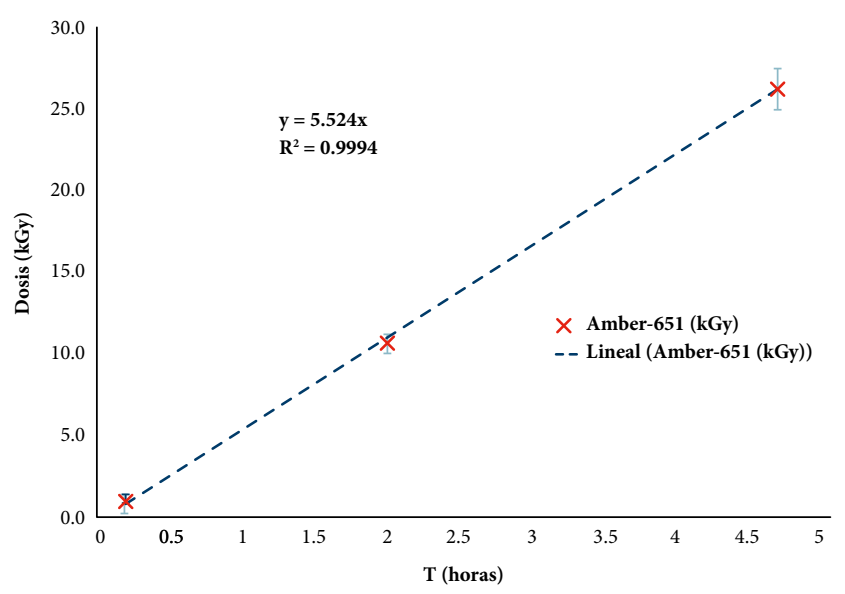

Gráfica 2. Dosis experimental contrastada con tiempo de irradiación en el punto establecido para el ensayo.

requerida para determinar experimentalmente los tiempos de dosis. Esta área es la misma descrita en el numeral 1.

En lo que respecta a la tasa de dosis obtenida experimentalmente en el punto físico definido a $15 \mathrm{~cm}$ de la barrera de aluminio y perpendicular al centro geométrico de la fuente, se procedió a irradiar tres dosímetros de rutina dentro del fantoma, para verificar los tiempos asociados a las dosis que se aplicarían y para cada sistema dosimétrico que se evaluaría, en los puntos $1 \mathrm{kGy}, 10 \mathrm{kGy}$ y $25 \mathrm{kGy}$, por triplicado.

Se determina la tasa de dosis promedio obtenida en una hora como producto de la medición de cada sistema dosimétrico de rutina por triplicado. Estos son los resultados obtenidos:

» Dosímetros Amber lote $\mathrm{X}=5,32 \pm 0,25 k G y / h$

$\gg$

Dosímetros Red lote $\mathrm{MH}=5,50 \pm 0,33 \mathrm{kG} y / \mathrm{h}$
Se grafica la dosis (kGy) contrastada con el tiempo (h) de los datos promedio de la tasa de dosis dada por los dosímetros tipo Amber, para estimar los tiempos de irradiación necesarios para alcanzar las dosis objetivo $\mathrm{x}_{\mathrm{pt}}$ (gráfica $2 \mathrm{y}$ tabla 1 ).

Tabla 1. Tiempos estimados de irradiación para las dosis objetivo xpt en el punto establecido para el ensayo.

\begin{tabular}{cccc}
\hline $\begin{array}{c}\text { Dosis objetivo } \\
(\mathbf{k G y})\end{array}$ & $\begin{array}{c}\text { Dosis promedio } \\
\text { Amber } \\
(\mathbf{6 5 1} \mathbf{~ n m})(\mathbf{k G y})\end{array}$ & cV\%* & $\begin{array}{c}\text { Tiempo estimado } \\
\text { de irradiación (h) }\end{array}$ \\
\hline 1 & 1,0 & 5,3 & 0,19 \\
\hline 5 & - & & 0,94 \\
\hline 10 & 10,7 & 4,3 & 1,88 \\
\hline 25 & 25,3 & 5,7 & 4,70 \\
\hline
\end{tabular}

$\overline{\mathrm{CV}} \%$ es el coeficiente de variación porcentual.

Estos tiempos son dados al operador para llevar a cabo la irradiación de las dosis objetivo $\mathrm{x}_{\mathrm{pt}}$.

Finalizado el proceso de irradiación, se evalúan las dosis irradiadas siguiendo el procedimiento descrito en el método de ensayo interno ME-TNU-PG, "Determinación de la dosis absorbida por dosimetría PMMA" [13].

Con los resultados obtenidos, presentados en la gráfica 2 , se determina si existe dosis de tránsito alguna por extrapolación al tiempo $t=0$ horas, causada por el paso de la fuente radiactiva a su posición izada, valor el cual resulta despreciable para el rango de dosis validado.

Se realiza la misma determinación con el sistema dosimétrico Red Perspex a $640 \mathrm{~nm}$ identificando un error por encima del 200\% ( $\mathrm{x}_{\mathrm{i}}>2 \mathrm{kGy}$ ) en una dosis teórica de 1 kGy. Este sistema dosimétrico no está validado para valores de dosis por debajo de $5 \mathrm{kGy}$; por tanto, se decide emplear como sistema dosimétrico para el ensayo el sistema Amber Perspex a $651 \mathrm{~nm}$.

\subsubsection{Análisis de las dosis de radiación gamma impartidas}

Se realizó el control de rutina al sistema espectrométrico de lectura. Se determinó la absorbancia específica y las dosis irradiadas en cada dosímetro, control llevado a cabo por dos dosimetristas, de manera aleatoria, el mismo día, al día siguiente, al quinto día, al sexto, octavo, hasta el decimosegundo día.

La evaluación estadística de los resultados experimentales presentados en la tabla 1 indica que la metodología de cálculo en las actividades definidas y descritas en el numeral 3 es correcta en las dosis objetivo $\mathrm{x}_{\mathrm{pt}}$. 
Se confirmó que el sistema Red Perspex no puede leer dosis tan bajas como $1 \mathrm{kGy}$ (da un valor más alto que el calculado), en contraste con el sistema Amber Perspex, que sí está validado para dicho valor de dosis.

\subsection{Irradiación en el sistema dosimétrico en el ensayo de aptitud}

Se procedió a irradiar los dosímetros de alanina, lote BF 616, junto con los dosímetros Amber Perspex 3042, lote X seleccionado.

Establecido el tiempo y posicionamiento del fantoma para irradiación del sistema dosimétrico de alanina, se identifica cada uno de los cinco pellets de alanina, cuatro con una de las dosis objetivo, y uno como "testigo", que no es irradiado.

Durante el proceso de irradiación individual se montan conjuntamente dentro del fantoma cuatro dosímetros de rutina Amber 3042 Perspex, lote X.

En cada uno de los ensayos se estima la temperatura de irradiación, midiendo la temperatura mínima de irradiación antes del inicio, y la temperatura máxima a la que llega el conjunto de dosímetros en el fantoma al final del proceso de irradiación.

\section{CÁLCULOS}

El programa de ensayo de aptitud fundamenta sus criterios de aceptación en lo establecido por la norma ISO/IEC 17043:2014, "Evaluación de la conformidad: Requisitos generales para ensayos de aptitud" [2].

Los parámetros que se deben determinar para el ejercicio ensayo de aptitud por comparaciones interlaboratorio para irradiación y medición de la dosis se subdividen en dos partes [3]:

Parte 1: Dosis de irradiación entregada a los dosímetros. Las dosis objetivo son $x_{p t}$ : 1 kGy, 5 kGy, 10 kGy y 25 kGy].

El evaluador del desempeño es el parámetro z-score:

$$
z_{i}=\frac{\left(x_{i}-x_{p t}\right)}{\sigma_{p t}}
$$

Donde:

》)

$x_{i}$ es el valor irradiado por el participante (obtenido por el Laboratorio de Dosimetría de Altas Dosis [LDAD] de la Comisión Nacional de Energía Atómica de Argentina de los dosímetros de alanina). $\mathrm{x}_{\mathrm{pt}}$ es el valor asignado (dosis dada: 1 kGy, 5 kGy, 10 kGy y 25 kGy)

» $\sigma_{p t}$ es la desviación estándar del ejercicio, según lo indicado en el anexo D.1.4 de la norma ISO 13528:2015 [14]

El criterio de aceptación [2] es:

»Si $|z| \leq 2$, el resultado se considera aceptable

\Si $2<|z|<3$, el resultado se considera cuestionable

» $\mathrm{Si}|z| \geq 3$, el resultado se considera no aceptable

Parte 2: Dosis de irradiación medida por cada participante

El evaluador del desempeño es el parámetro $\mathrm{E}_{\mathrm{n}}$ :

$$
\left(E_{n}\right)_{i}=\frac{\left(x_{i}-x_{p t}\right)}{\sqrt{U^{2}\left(x_{i}\right)+U^{2}\left(x_{p t}\right)}}
$$

Donde:

» $x_{i}$ es el valor reportado por cada laboratorio a partir de su dosimetría de rutina

» $x_{p t}$ es el valor asignado, igual al obtenido por el LDAD (en la ecuación 1 , este valor equivale a $\mathrm{x}_{\mathrm{i}}$ )

》 $U\left(x_{i}\right)$ es la incertidumbre expandida del valor $\mathrm{x}_{\mathrm{i}}$

» $U\left(x_{p t}\right)$ es la incertidumbre expandida del valor asignado obtenido por el LDAD

El criterio de aceptación [2] es:

》S $\mathrm{Si}\left|E_{n}\right|<1$, el resultado se considera aceptable

» $\mathrm{Si}\left|E_{n}\right| \geq 1$, el resultado se considera no aceptable

\subsection{Estimación de la incertidumbre}

Para estimar la incertidumbre del ensayo de aptitud se siguió estrictamente la metodología conocida como botttom up o componente por componente [15], [16], que se enfoca en una serie de pasos sistemáticos, donde el responsable de la estimación descompone todas las operaciones (analíticas-operativas) en actividades primarias. Seguidamente se combinan o agrupan en actividades comunes y se hace una estimación de su contribución al valor combinado de incertidumbre del proceso de medición (incertidumbre relativa - $\mu$ ) [15], [17]. Estas fuentes se combinan para dar como resultado una incertidumbre expandida (U), [15], [17].

Este método exige un conocimiento exacto de todo el proceso, tanto analítico como operativo. Su principal ventaja 
es que proporciona una clara comprensión de las actividades analíticas que suponen una importante contribución a la incertidumbre de la medición y que, por consiguiente, pueden asignarse como puntos críticos de control para reducir o dirigir la estimación de la incertidumbre de la medición en las aplicaciones futuras para el proceso de dosimetría de rutina.

Se enuncian las principales contribuciones identificadas y su respectivo peso en la estimación de la incertidumbre para el punto a $x_{p t}=25 \mathrm{kGy}$ :

\subsubsection{Incertidumbre tipo A}

a. Variabilidad del método (precisión intermedia). Aporta el $18,9 \%$ de la $\mu$.

b. Variabilidad del tiempo de la lectura posirradiación de los dosímetros (precisión intermedia). Aporta el 25,1\% de la $\mu$.

c. Variabilidad del espesor del dosímetro (precisión intermedia). Aporta el 4,8\% de la $\mu$.

\subsubsection{Incertidumbre tipo $B$}

a. Precisión del espectrofotómetro (longitud de onda). Aporta el 1,5\% de la $\mu$.

b. Precisión del micrómetro. Aporta el 0,2\% de la $\mu$.

c. Cambio de temperatura del dosímetro. Aporta el 11,6\% de la $\mu$. La incertidumbre de la variación de la temperatura se considera como la variación reportada por el conjunto del fantoma y los dosímetros, y se le asigna una distribución rectangular.

d. Coeficiente de expansión térmica por diferencia de temperaturas del dosímetro. Aporta el 0,001\% de la $\mu$. La incertidumbre del coeficiente de expansión térmica en diferentes materiales se estima como un $10 \%$ del valor del coeficiente, y se le asigna una distribución rectangular. El coeficiente del PMMA es 77 x 10-6 $\left({ }^{\circ} \mathrm{K}^{-1}\right)$. Se determina con la expresión de la expansión térmica lineal de los cuerpos.

e. Interpolación en la curva de calibración. Aporta el $37,8 \%$ de la $\mu$.

La variabilidad del método y la variabilidad del tiempo de medición posirradiación de los dosímetros, que corresponden concretamente a las actividades propias analíticas del ensayo por espectrometría visible, y la interpolación de la dosis irradiada en la curva del sistema dosimétrico PMMA, representan la mayor contribución en el aporte a la estimación de la incertidumbre.

Esto quiere decir que el punto de control de mayor rigurosidad y atención se encuentra en el proceso de dosimetría de rutina. Aquí el término control hace referencia a las actividades realizadas que permitirán asegurar la calidad de los resultados de las mediciones dosimétricas de rutina, enmarcadas en un sistema de gestión.

\section{RESULTADOS Y ANÁLISIS}

Se da cumplimiento al protocolo establecido por INTERLAB: cada uno de los dosímetros de alanina es irradiado individualmente en las dosis objetivo, en las condiciones propias de operación de la instalación, junto con cuatro dosímetros del sistema de rutina empleado por la Planta de Irradiación Gamma (dosímetro Amber 3042 Perspex). Los rangos de temperatura no superaron los $1,1^{\circ} \mathrm{C}$ y se mantuvieron en los valores recomendados para el sistema PMMA [12].

Los resultados de dosis aplicadas a los sistemas dosimétricos de ensayo y reportadas a INTERLAB, así como los resultados de los parámetros de estudio dados en [3], se presentan en la tabla 2.

La calificación final corresponde al contraste de los parámetros calculados en el modelo para el tratamiento estadístico planteado por INTERLAB con los criterios de aceptación mencionados en el numeral anterior, a partir de lo cual se eligen como evaluadores de desempeño los parámetros z (z-score), que corresponden al sesgo del resultado respecto al valor de referencia, $\mathrm{y}_{\mathrm{n}}$, que corresponde a la evaluación de la incertidumbre estimada.

Se observa que todos los valores de los parámetros de desempeño evaluados en cada dosis impartida están en el rango determinado por los criterios de aceptación; por lo tanto, la calificación final para la Planta de Irradiación Gamma es satisfactoria o aceptable.

En la tabla 3 se tabula el porcentaje de laboratorios participantes que obtuvieron calificación satisfactoria en cada una de las dosis objetivo.

Los porcentajes evidencian el rigor del ejercicio y la capacidad del Laboratorio de Dosimetría de Rutina de la Planta de Irradiación para reportar los datos de dosis, con una incertidumbre que se mantiene en el rango de desempeño aceptable en todas las dosis objetivo del ejercicio. Es evidente la mayor dificultad para obtener un valor de $\mathrm{E}_{\mathrm{n}}$ en el rango 
aceptable en todos los laboratorios participantes; de hecho, para 5 kGy, la Planta de Irradiación obtuvo valores en el rango, y fue una de las que se mantuvieron en el estrecho margen del 33\% de participantes que lo lograron.

\section{CONCLUSIONES}

La evaluación de la medida de desempeño presentada por INTERLAB correspondiente a los resultados entregados por la Planta de Irradiación Gamma, en donde se calificaron dos parámetros de desempeño, $\mathrm{z}$ ( $\mathrm{z}$-score) y $\mathrm{E}_{\mathrm{n}}$, en las cuatro dosis objetivo definidas en el ensayo de aptitud, es aceptable. Este resultado califica el desempeño de la Planta de Irradiación Gamma relacionado con el proceso de irradiación, la competencia del personal y la garantía de la calidad analítica en los más altos estándares de calidad.

La robustez del procedimiento empleado para estimar la incertidumbre es una pieza fundamental en la implementación del sistema de calidad basado en la norma ISO 11137, asociado a la validación del proceso de operación, el proceso de dosimetría de rutina, la calificación de la instalación y la calificación del desempeño

Se obtuvieron diferencias entre el valor medido y el valor asignado como referencia menores del 10\% (gráfica 3), que se considera un buen resultado [3].

Se debe continuar participando en la realización de más ensayos de aptitud para mantener el sistema de gestión de calidad y verificar la efectividad de las acciones de mejora tomadas.

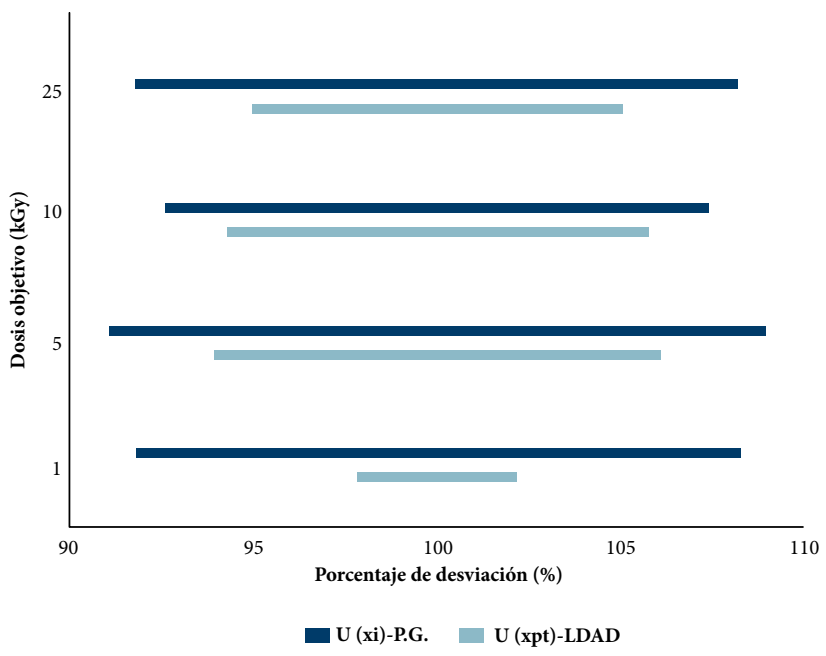

Gráfica 3 Resultados de dosis e incertidumbres logradas por los sistemas dosimétricos de rutina PMMA de la Planta de Irradiación Gamma (P. G.) y el de referencia del Laboratorio de Dosimetría de Altas Dosis (LDAD).

\section{Agradecimientos}

Los autores agradecen a Miguel Antonio Vargas y Gelfi Nelson Beltrán, funcionarios del grupo de Geoquímica Analítica y Geotecnia de la Dirección de Laboratorios, y al antiguo operador de la Planta de Irradiación, Jaime Bravo, del Servicio Geológico Colombiano, por su ayuda en la construcción y adaptación del fantoma utilizado en este ejercicio.

Gracias también a Andrea Docters, jefe de la Planta de Irradiación de la CNEA, por su colaboración en la gestión de los dosímetros de referencia a ser entregados al LDAD.

Tabla 2. Resultados finales, Planta de Irradiación Gamma (P. G.) vs. Laboratorio de Dosimetría de Altas Dosis (LDAD)

\begin{tabular}{|c|c|c|c|c|c|c|c|c|c|}
\hline $\begin{array}{c}\text { Dosis objetivo } \\
\text { (kGy) }\end{array}$ & $\begin{array}{c}\text { Temperatura } \\
\text { inicial de } \\
\text { irradiación }\left({ }^{\circ} \mathrm{C}\right)\end{array}$ & $\begin{array}{l}\text { Temperatura } \\
\text { final de } \\
\text { irradiación }\left({ }^{\circ} \mathrm{C}\right)\end{array}$ & $\begin{array}{l}\text { Dosis PMMA P. } \\
\text { G. (kGy) }\end{array}$ & $\begin{array}{l}\text { U PMMA P. G. } \\
(\mathrm{k}=2)\end{array}$ & $\begin{array}{c}\text { Dosis alanina } \\
\text { LDAD } \\
(\mathrm{kGy})\end{array}$ & $\begin{array}{c}\text { U alanina } \\
\text { LDAD }(k=2)\end{array}$ & $\begin{array}{c}\text { Parámetro } \mathrm{z} \\
\text { (z-score) }\end{array}$ & Parámetro En & Calificación \\
\hline 1 & 26,6 & 26,2 & 0,99 & 0,08 & 0,99 & 0,02 & $-0,09$ & 0,06 & Aceptable \\
\hline 5 & 25 & 25,5 & 4,89 & 0,43 & 4,73 & 0,28 & $-0,55$ & 0,38 & Aceptable \\
\hline 10 & 23,5 & 24,6 & 10,22 & 0,74 & 9,47 & 0,53 & $-0,58$ & 0,99 & Aceptable \\
\hline 25 & 24,9 & 24,7 & 24,54 & 1,98 & 23,83 & 1,17 & $-1,07$ & 0,34 & Aceptable \\
\hline
\end{tabular}

Tabla 3. Porcentaje de laboratorios participantes que obtuvieron calificaciones aceptables o cuestionables en z-score y aceptables en En

\begin{tabular}{cccc}
\hline Dosis objetivo (kGy) & Porcentaje en el rango aceptable (z) & Porcentaje en el rango cuestionable (z) & Porcentaje en el rango aceptable (En) \\
\hline 1 & $92 \%$ & $0 \%$ & $67 \%$ \\
\hline 5 & $92 \%$ & $0 \%$ & $0 \%$ \\
\hline 10 & $92 \%$ & $5 \%$ & $5 \%$ \\
\hline 25 & $85 \%$ & $55 \%$ & \\
\hline
\end{tabular}




\section{Referencias}

[1] ONAC, "Ensayo de aptitud", 2019. [Online]. Disponible en https://onac.org.co/proveedores-de-ensayos-de-aptitud.

[2] ISO/IEC, "ISO/IEC 17043:2014 Evaluación de la conformidad: Requisitos generales para ensayos de aptitud", 2014. Disponible en: https://www.iso.org/obp/ui\#iso:std:iso-iec:17043:ed-1:v1:es

[3] INTERLAB y CNEA, "Informe final N-DO-02. Ensayo de aptitud por comparaciones interlaboratorio en irradiación y medición de dosímetros", Buenos Aires, 2019.

[4] H. Garonis, F. di Giacomo, D. Russo y E. Camacho, "Proceso de realización de los ensayos de aptitud por comparación interlaboratorio". En Simposio de Metrología, 2010, p. 5.

[5] ISO, "ISO 9001:2015 - Quality management systems: Requirements", p. 29, 2015.

[6] ISO/IEC, "ISO/IEC 17025:2005 - General requirements for the competence of testing and calibration laboratories", p. 28, 2005.

[7] CNEA, "Interlaboratorios e Instalaciones, Interlab", 2019.

[8] ONAC, "Acuerdos de reconocimiento internacional ONAC". [Online]. Disponible en https://onac.org.co/ acuerdos-de-reconocimiento-internacional.
[9] International Atomic Energy Agency, Radiation safety of gamma, electron and $X$ ray irradiation facilities, IAEA SAFET. Vienna: IAEA, 2010.

[10]ISO/IEC, "ISO/ASTM 51276:2019 (en). Practice for use of a polymethylmethacrylate dosimetry system", 2019.

[11]ISO/IEC, "ISO/ASTM 51261:2013 (en). Practice for calibration of routine dosimetry systems for radiation processing", p. 18, 2013.

[12]B. Whittaker y M. F. F. Watts, "The influence of dose rate, ambient temperature and time on the radiation response of Harwell PMMA dosimeters", Radiation Physics and Chemistry, vol. 60, n. ${ }^{\circ} 1-2$, pp. 101-110, 2001. https://doi.org/10.1016/S0969-806X(00)00316-9

[13]J. I. Abril, "Determinación de la dosis absorbida por dosimetría PMMA", Bogotá, D. C., 2017.

[14]ISO/IEC, "ISO/IEC 13528:2015, Estatisticals methods for use in proficiency testing by laboratory comparisons", 2015.

[15] BIPM, GUM - Evaluation of measurement data: Guide to the expression of uncertainty in measurement, $1^{\circ}$. Sevres: JCGM, 2008.

[16] Eurachem/CITAC, Cuantificación de la incertidumbre en medidas analíticas: Guía CITAC 4, 3. ${ }^{\mathrm{a}}$ ed. UK: UK National Measurement System, 2011.

[17]BIPM, VIM - International Vocabulary of Metrology: Basic and general concepts and associated terms, $3 .^{\circ}$. Sevres: JCGM, 2008. 